\title{
Two-color QCD in 3D at finite baryon density
}

\author{
Gerald V. Dunne and Shinsuke M. Nishigaki \\ Department of Physics, University of Connecticut, Storrs, CT 06269-3046, USA
}

(Dated: October 15, 2002)

\begin{abstract}
We study the low energy phase structure of $\mathrm{SU}(2)$ gauge theories in three-dimensional spacetime, at finite baryon density. The pseudoreality of representations of $\mathrm{SU}(2)$ permits an analytic study of a real baryon chemical potential, and the restriction to 3D results in a different global symmetry breaking pattern from the corresponding 4D model studied previously by Kogut et al. We find a second-order phase transition separating the normal phase and the baryon superconducting phase. The chemical potential dependence of condensates and baryon density are computed. We find that the phase structure and the excitation spectrum are essentially the same as in $4 \mathrm{D}$, despite the different symmetry groups, indicating a universality that is rooted in the properties of Riemannian symmetric spaces.
\end{abstract}

PACS numbers: $11.10 . \mathrm{Kk}, 11.30 . \mathrm{Hv}, 11.30 . \mathrm{Qc}, 12.39 . \mathrm{Fe}$

Keywords: flavor symmetry, chiral Lagrangian, chemical potential, baryon superconductivity

\section{INTRODUCTION}

The spontaneous breaking of global symmetries is an essential feature of realistic gauge theories [1]. Much has been learned about this phenomenon by studying models in various dimensions. For example, the 2D Schwinger model and 4D QCD exhibit the breakdown of discrete or continuous chiral symmetry, respectively. For even dimensional spacetime there is a comprehensive theorem that predicts the surviving part of of the flavor symmetry [2]. For theories in odd dimensional spacetime the situation is rather different, primarily because there is no chirality and the parity transformation acts differently [3]. In particular, models in 3D spacetime, while different from 4D theories, provide excellent testing grounds for our understanding of dynamical symmetry breaking. Yet even here the picture is still not completely clear. The generic symmetry breaking pattern in 3D has been described for Abelian theories in [4]. In large $N_{F} \mathrm{QED}_{3}$ there are indications of spontaneous breaking of the global flavor symmetry [5, 6]. Studies of the Schwinger-Dyson and gap equations for $\mathrm{QED}_{3}$ in the large $N_{F}$ limit predict that spontaneous symmetry breaking only occurs for $N_{F}$ below a critical number of flavors $N_{F}^{\text {crit }}=32 / \pi^{2} \approx 3[\underline{7},[]$ ] . These predictions have numerical support from lattice simulations of 3D QED 20]. On the other hand, other 3D QED Schwinger-Dyson analyses [10] predict symmetry breaking for all $N_{F} \geq 2$, a result which is consistent with the renormalization group arguments in 11].

Another approach to the symmetry breaking structure of fermion-gauge theories is to use low-energy effective Lagrangians 12, 13] to study the Goldstone modes corresponding to the spontaneously broken global symmetries. Recently, there has been a great deal of progress (for a review see 14]) in using such low-energy effective Lagrangians, together with random matrix models, to study the phase structure of nontrivial QCD-like theories with spontaneous symmetry breaking. An important obstacle, however, is that it is not known how to study these systems at finite baryon density, since the baryon number chemical potential makes the Euclidean Dirac operator non-Hermitean and the Boltzmann weight complex. This problem can be overcome, as advocated in [15, 16, 17, 18, 19], by considering "two-color QCD", for which the fundamental representation of $\mathrm{SU}(2)$ is pseudoreal. This has the consequence that in lattice simulations the Boltzmann weight is real and positive definite, even at finite (baryon number) chemical potential $\mu$. Thus, analytic predictions can be quantitatively compared with Monte Carlo simulations in lattice gauge theory [20, 21], provided the lattice regularization respects the relevant flavor symmetry group. Such studies have recently been carried out 22] in 4D QCD with quarks in pseudoreal (and real) representations at finite $\mu$.

In this paper we study the phase structure of such $\mathrm{SU}$ (2) QCD-like theories in 3D, at finite baryon density. The possibility of using a real chemical potential still applies in 3D. Our strategy is analogous to the 4D analysis of Kogut, Stephanov, Toublan, Verbaarschot and Zhitnitsky 17] (denoted KSTVZ hereafter), but the details of the symmetry breaking patterns are completely different in $3 \mathrm{D}$ compared to $4 \mathrm{D}$. However, we find that the final answer is almost identical to the 4D case, indicating some degree of universality in the low energy vacuum phase structure. This low energy effective Lagrangian approach assumes that the symmetry breaking occurs, so it is not able to resolve a question such as the existence of a critical number of flavors. Nevertheless, we hope that our results may shed some light on the phase structure of the $3 \mathrm{D}$ theories, for example in conjunction with lattice analyses.

In 3D, with an even number $N_{F}$ of flavors of massless complex fermions, denoted by the $N_{F} / 2$ pairs $\psi_{f}, \chi_{f}$, one can predict spontaneous flavor symmetry breaking patterns along similar lines to $4 \mathrm{D}$ QCD. In 3D one can introduce of a fermion mass term

$$
\mathcal{L}_{m}=\sum_{f=1}^{N_{F} / 2} m_{f}\left(\bar{\psi}_{f} \psi_{f}-\bar{\chi}_{f} \chi_{f}\right) .
$$


which preserves a discrete symmetry that is a combination of parity and flavor exchange. The fermion determinant $\prod_{f=1}^{N_{F} / 2} \operatorname{det}\left(-\not D^{2}+m_{f}^{2}\right)$ for Euclidean space (where the Dirac operator $\not D$ is anti-Hermitian) is positive definite in this case. Therefore one can appeal to the VafaWitten theorem 2] and predict that if the flavor symmetry is spontaneously broken in the limit $m_{f} \equiv m \rightarrow 0$, the absolute values of the condensates $\left\langle\bar{\psi}_{f} \psi_{f}\right\rangle$ and $\left\langle\bar{\chi}_{f} \chi_{f}\right\rangle$ are equal and their signs are the same as those of respective masses. That is, the generic situation is that the continuous part of the global symmetry group is broken according to [4, 5 .

$$
\mathrm{U}\left(N_{F}\right) \rightarrow \mathrm{U}\left(N_{F} / 2\right) \times \mathrm{U}\left(N_{F} / 2\right)
$$

by the quark-antiquark condensate

$$
\sum_{f=1}^{N_{F} / 2}\left(\left\langle\bar{\psi}_{f} \psi_{f}\right\rangle-\left\langle\bar{\chi}_{f} \chi_{f}\right\rangle\right),
$$

Evidence for such a symmetry breaking pattern has been observed in 3D lattice simulations 23] with gauge group $\mathrm{SU}(3)$. This pattern of flavor symmetry breaking can also be predicted for $3 \mathrm{D}$ QCD at large $N_{C}$ using the Coleman-Witten argument 24].

The 3D symmetry breaking pattern in (2) is for quarks in a complex representation of the gauge group, and is expected to apply to a $\mathrm{U}(1)$ theory and to $\mathrm{SU}\left(N_{C}\right)$ theories with number of colors $N_{C} \geq 3$. For $\mathrm{SU}(2)$, with fundamental quarks, the symmetry breaking pattern is expected to be different again, due to the pseudoreality of the fundamental representation. The pseudoreality of the fundamental representation of $\mathrm{SU}(2)$ means that the naive $\mathrm{U}\left(N_{F}\right)$ flavor symmetry is extended to $\operatorname{USp}\left(2 N_{F}\right)$, and the continuous part of this global symmetry group is predicted to break down in 3D as 25]

$$
\mathrm{USp}\left(2 N_{F}\right) \rightarrow \operatorname{USp}\left(N_{F}\right) \times \operatorname{USp}\left(N_{F}\right) .
$$

This is different from the generic 3D symmetry breaking pattern in (2), and also is different from the symmetry breaking patterns in $4 \mathrm{D}$ theories where the standard flavor symmetry breaking patterns are

$$
\begin{aligned}
\mathrm{U}\left(N_{F}\right)_{L} \times \mathrm{U}\left(N_{F}\right)_{R} \rightarrow \mathrm{SU}\left(N_{F}\right)_{V}, & \text { for } \mathrm{SU}\left(N_{C} \geq 3\right) \\
\mathrm{U}\left(2 N_{F}\right) \rightarrow \mathrm{USp}\left(2 N_{F}\right), & \text { for } \mathrm{SU}(2)
\end{aligned}
$$

In $4 \mathrm{D}$ the $\mathrm{U}\left(N_{F}\right)$ flavor symmetries are first broken to $\mathrm{SU}\left(N_{F}\right)$ symmetries by the axial anomaly, and then broken by the chiral condensate, with the net breakings as shown in (5).

Physically, the differences between the $3 \mathrm{D}$ and $4 \mathrm{D}$ cases reflect the differences between the anomalous discrete symmetries of parity and chirality in $3 \mathrm{D}$ and $4 \mathrm{D}$, respectively. The differences between the gauge groups $\mathrm{SU}(2)$ and $\mathrm{SU}\left(N_{C} \geq 3\right)$ are due to the properties of the representations of these groups. From studies of $4 \mathrm{D}$ theories, it has long been appreciated that the $\mathrm{SU}(2)$ theory exhibits exotic types of spontaneous breakdown of global symmetry [26]. Because the representation of the $\mathrm{SU}(2)$ gauge group is either pseudoreal or real, quarks and charge-conjugated antiquarks are combined into an extended flavor multiplet, which is expected to break into its extended vector subgroup. These arguments are actually sensitive only to the representation properties of the gauge group as long as the theory confines. The difference between $\mathrm{SU}(2)$ and $\mathrm{SU}\left(N_{C} \geq 3\right)$ can also be understood in terms of Witten's SU(2) anomaly in 4D 27] and its $3 \mathrm{D}$ counterpart 28 .

In this paper we study the 3D $\mathrm{SU}(2)$ theory, with fundamental quarks, in order to be able to include a real baryon number chemical potential $\mu$. We follow closely the strategy and analysis of KSTVZ, where the $4 \mathrm{D} \mathrm{SU}(2)$ system with symmetry breaking pattern $\mathrm{U}\left(2 N_{F}\right) \rightarrow \mathrm{USp}\left(2 N_{F}\right)$, as in (5), has been analyzed at finite chemical potential. Since the $3 \mathrm{D} \mathrm{SU}(2)$ theory is predicted to have symmetry breaking pattern $\operatorname{USp}\left(2 N_{F}\right) \rightarrow$ $\mathrm{USp}\left(N_{F}\right) \times \operatorname{USp}\left(N_{F}\right)$, as in (4), the low-energy degrees of freedom are completely different. Clearly, this means that the nonlinear $\sigma$ model that describes the Goldstone bosons associated with the flavor symmetry breaking is different from the 4D case studied in KSTVZ.

Since the key feature of the SU(2) gauge theory that permits a real chemical potential is the existence of the pseudoreal fundamental representation, it is clear that these ideas apply also to any gauge theory with pseudoreal quarks, namely $\mathrm{USp}\left(2 N_{C}\right)$ gauge theory with fundamental fermions. A complementary case with real quarks, i.e. $\mathrm{SO}\left(N_{C}\right)$ gauge theories with fundamental fermions, and $\mathrm{SU}\left(N_{C} \geq 2\right)$ gauge theories with adjoint fermions, was also studied at finite chemical potential in $4 \mathrm{D}$ in KSTVZ, and yielded a result universal to the case with pseudoreal quarks. In $3 \mathrm{D}$ the naive $\mathrm{U}\left(N_{F}\right)$ flavor symmetry of the theories with real fermions is extended to $\mathrm{O}\left(2 N_{F}\right)$, and the predicted flavor symmetry breaking pattern is [29]

$$
\mathrm{O}\left(2 N_{F}\right) \rightarrow \mathrm{O}\left(N_{F}\right) \times \mathrm{O}\left(N_{F}\right) .
$$

Since the pattern of symmetry breaking is different, it is clear that the nonlinear $\sigma$ model that describes the Goldstone bosons associated with the flavor symmetry breaking is different. For the generic 3D flavor breaking in (2) the nonlinear $\sigma$ model has a complex Grassmannian as its target space manifold, while for the pseudoreal fermion case (4) and the real fermion case (6) the $\sigma$ model target space is a quaternionic and real Grassmannian, respectively. Despite these distinctions between pseudoreal and real fermions, and between the $3 \mathrm{D}$ and $4 \mathrm{D}$ cases, we shall show in this paper that the vacuum condensates and the dispersion laws of the low-lying excitations share the same functional dependences on the chemical potential.

In section II we briefly review the symmetry breaking pattern in 3D for gauge theories with quarks in pseudoreal and real representations, at zero and finite chemical potential. In section III we construct the corresponding low energy effective Lagrangians by global and local flavor symmetry arguments. In section IV we analyze the 
phase structure of the models by finding the minimum of the effective potential, and compute the condensate and the baryon density. In sections V and VI we expand the potential and kinetic terms, respectively, of the effective Lagrangian to quadratic order and derive dispersion laws for the excitations. In section VII we conclude by providing a group theoretic account for the observed universality.

\section{GLOBAL SYMMETRY}

\section{A. Enlarged flavor symmetry}

The fermionic kinetic part of the two-color QCD Lagrangian with $N_{F}=2 n$ flavors of quarks in Euclidean $3 \mathrm{D}$ space is given by

$$
\mathcal{L}_{\text {kin }}=\bar{\psi} \not D \psi+\bar{\chi} \not D \chi
$$

Here $\psi=\psi_{f}^{i}, \psi^{\dagger}=\psi_{f}^{* i}, \chi=\chi_{f}^{i}, \chi^{\dagger}=\chi_{f}^{* i}$ are independent two-component spinor fields, with the color index $i=1,2$ and flavor index $f=1, \ldots, n$ being suppressed. Pauli matrices denoted as $\sigma_{\nu}$, with $\nu=1,2,3$, are employed to represent the Euclidean Dirac matrix algebra, and those denoted as $\tau_{\alpha}$, with $\alpha=1,2,3$, are employed to represent the gauge group algebra. We choose $x_{3}$ to be the Euclidean time direction and define $\bar{\psi}=\psi^{\dagger} \sigma_{3}$, $\bar{\chi}=\chi^{\dagger} \sigma_{3}$. The Dirac operator is $\not D=\sigma_{\nu} D_{\nu}$, and the covariant derivative is $D_{\nu}=\partial_{\nu}+i A_{\nu}$, and the gauge field $A_{\nu}=A_{\nu}^{\alpha} \tau_{\alpha}$ is Hermitian and $\mathrm{su}(2)$ valued.

We take the parity transformation $(\mathrm{P})$ to be a reflection in the $x_{1}$ direction,

$$
x=\left(x_{1}, x_{2}, x_{3}\right) \mapsto x_{P}=\left(-x_{1}, x_{2}, x_{3}\right) .
$$

Its actions on the fermion fields are [3]

$$
\psi(x) \mapsto \sigma_{1} \psi\left(x_{P}\right), \quad \bar{\psi}(x) \mapsto-\bar{\psi}\left(x_{P}\right) \sigma_{1} .
$$

The flavor $\mathbf{Z}_{2}$ transformation is an exchange of $\psi$ and $\chi$,

$$
\psi(x) \leftrightarrow \chi(x), \quad \bar{\psi}(x) \leftrightarrow \bar{\chi}(x) .
$$

We require the fundamental Lagrangian to be invariant under the combination of these two transformations, which we call the $\left(\mathrm{P}, \mathbf{Z}_{2}\right)$-symmetry. Clearly the kinetic term is $\left(\mathrm{P}, \mathbf{Z}_{2}\right)$-invariant.

Due to the pseudoreality of the SU(2) Dirac operator, the Lagrangian (7) is invariant under a symmetry group larger than the apparent $\mathrm{U}(2 n)$. Under the gauge transformation $\psi \rightarrow g \psi, \bar{\psi} \rightarrow \bar{\psi} g^{\dagger}, g \in \mathrm{SU}(2)$ and the Lorentz transformation $\psi \rightarrow u \psi, \bar{\psi} \rightarrow \bar{\psi} u^{\dagger}, u \in \mathrm{SU}(2)$, a combination $\tilde{\psi}=\sigma_{2} \tau_{2} \bar{\psi}^{T}$ transforms as $\tilde{\psi} \rightarrow g \tilde{\psi}$ and $\tilde{\psi} \rightarrow u \tilde{\psi}$. Thus one can put $\psi, \chi, \tilde{\psi}$, and $\tilde{\chi}$ into a single flavor $4 n$-plet,

$$
\Psi=\left[\begin{array}{c}
\psi \\
\chi \\
\sigma_{2} \tau_{2} \bar{\psi}^{T} \\
\sigma_{2} \tau_{2} \bar{\chi}^{T}
\end{array}\right]
$$

so that

$$
\mathcal{L}_{\text {kin }}=\frac{1}{2} \Psi^{T} \sigma_{2} \tau_{2} \not D I \Psi, \quad I=\left[\begin{array}{l|l} 
& -\mathbb{1}_{2 n} \\
\hline \mathbb{1}_{2 n} &
\end{array} .\right.
$$

As the products of the Pauli matrices $\sigma_{2} \sigma_{\nu}$ and $\tau_{2} \tau_{\alpha}$ are symmetric, so is the operator $\sigma_{2} \tau_{2} \not D$. Accordingly the matrix acting on flavor indices that appears in the above fermion bilinear has to be antisymmetric. In this form the extended flavor symmetry group is manifestly the unitary symplectic group $\operatorname{USp}(4 n)$,

$$
\Psi \rightarrow S \Psi, \quad S^{T} I S=I, \quad S^{\dagger} S=\mathbb{1}_{4 n} .
$$

The above extension of the flavor symmetry group is analogous to the two-color QCD in 4D where the conjugated right-handed spinor $\sigma_{2} \tau_{2} \psi_{R}^{*}$ transforms as the lefthanded spinor $\psi_{L}$ does under gauge and Lorentz transformations, so that the chiral $\mathrm{SU}\left(N_{F}\right)_{L} \times \mathrm{SU}\left(N_{F}\right)_{R}$ symmetry gets extended to $\mathrm{SU}\left(2 N_{F}\right)$.

\section{B. Mass term}

The $\left(\mathrm{P}, \mathbf{Z}_{2}\right)$-invariant bare mass term with degenerate masses,

$$
\mathcal{L}_{m}=m(\bar{\psi} \psi-\bar{\chi} \chi)
$$

can be rewritten as

$$
\mathcal{L}_{m}=\frac{m}{2} \Psi^{T} \sigma_{2} \tau_{2} \hat{M} \Psi, \quad \hat{M}=\left[\begin{array}{ll|ll} 
& & -\mathbb{1}_{n} & \\
& & \mathbb{1}_{n} \\
\hline \mathbb{1}_{n} & & \\
& -\mathbb{1}_{n} &
\end{array}\right] .
$$

The flavor group $\operatorname{USp}(4 n)$ is broken down to $\operatorname{USp}(2 n) \times$ $\operatorname{USp}(2 n)$ each acting on the (1,3)- and (2,4)-block,

$\Psi \rightarrow S \Psi, \quad S=\left[\begin{array}{ll|ll}s_{1}^{11} & & s_{1}^{12} & \\ & s_{2}^{11} & & s_{2}^{12} \\ \hline s_{1}^{21} & & s_{1}^{22} & \\ & s_{2}^{21} & & s_{2}^{22}\end{array}\right], \quad s_{1}, s_{2} \in \operatorname{USp}(2 n)$,

explicitly by this mass term, or spontaneously by the quark-antiquark condensate (3) if formed. In the latter case, the Goldstone manifold is thus given by a quaternionic Grassmannian $\operatorname{USp}(4 n) /(\operatorname{USp}(2 n) \times \operatorname{USp}(2 n))$. It has $4 n^{2}$ independent degrees of freedom, twice the three-color case $2 n^{2}$ of the complex Grassmannian manifold $\mathrm{U}(2 n) /(\mathrm{U}(n) \times \mathrm{U}(n))$. The Grassmannian $\operatorname{USp}(4 n) /(\mathrm{USp}(2 n) \times \mathrm{USp}(2 n))$ can be canonically parametrized as

$$
\Sigma=S \Sigma_{c} S^{T}, \quad \Sigma_{c}=\hat{M}
$$

where

$$
S(x)=\exp \left(\frac{i \Pi(x)}{2 F}\right), \quad \Pi(x)=\pi_{a}(x) X_{a} .
$$


The fields $\pi_{a}$ are the Goldstone modes, and the $4 n^{2}$ generators $X_{a}$ span the subspace $\operatorname{usp}(4 n)-(\operatorname{usp}(2 n) \oplus$ $\operatorname{usp}(2 n))$. The construction of the Goldstone manifold (18) corresponds to the classification of the generators of $\operatorname{usp}(4 n)$ with respect to a fixed antisymmetric matrix $\Sigma_{c}$ into $T_{k}$ and $X_{a}$; The $T_{k}$ generators that span $\operatorname{usp}(2 n) \oplus \operatorname{usp}(2 n)$ leave $\Sigma_{c}$ invariant,

$$
\mathrm{e}^{i \phi_{k} T_{k}} \Sigma_{c}\left(\mathrm{e}^{i \phi_{k} T_{k}}\right)^{T}=\Sigma_{c} \text {, i.e. } T_{k} \Sigma_{c}=-\Sigma_{c} T_{k}^{T} .
$$

The remaining generators $X_{a}$ obey the relations

$$
X_{a} \Sigma_{c}=\Sigma_{c} X_{a}^{T}, \text { i.e. } S \Sigma_{c} S^{T}=S^{2} \Sigma_{c} .
$$

The above partition of the $\operatorname{usp}(4 n)$ generators depends on the matrix $\Sigma_{c}$. The defining relations (20) are left unaltered by rotation of $\Sigma_{c}$ according to

$$
\Sigma_{c} \rightarrow s \Sigma_{c} s^{T}, \quad s \in \mathrm{USp}(2 n)
$$

accompanied by a simultaneous rotation of the generators by

$$
X_{a} \rightarrow s X_{a} s^{\dagger}
$$

This means that the set of broken generators, $X_{a}$, changes if the matrix $\Sigma_{c}$ is changed. The choice of $\Sigma_{c}=\hat{M}^{\dagger}$ leads to the block representation of the generators $X_{a}$ as

$$
\Pi=\frac{1}{2}\left[\begin{array}{c|cc}
P^{\dagger} & Q^{T} & \\
\hline Q^{\dagger} & Q^{*} & -P^{T}
\end{array}\right],
$$

where $P$ and $Q$ are $n \times n$ complex matrices, each having $2 n^{2}$ degrees of freedom.

\section{Chemical potential}

The chemical potential that coupled to the baryon number does not discriminate between the flavors $\psi$ and $\chi$, and is given by the $\left(\mathrm{P}, \mathbf{Z}_{2}\right)$-invariant form

$$
\mathcal{L}_{\mu}=-\mu\left(\psi^{\dagger} \psi+\chi^{\dagger} \chi\right)
$$

Without loss of generality we take $\mu \geq 0$. It can be rewritten in terms of the $4 n$-flavored spinor $\Psi$ defined in (11), as

$$
\mathcal{L}_{\mu}=-\frac{\mu}{2} \Psi^{T} i \sigma_{1} \tau_{2} \hat{C} \Psi, \quad \hat{C}=\left[\begin{array}{l|l} 
& \mathbb{1}_{2 n} \\
\hline \mathbb{1}_{2 n} &
\end{array}\right]
$$

This chemical potential term explicitly breaks the extended flavor group $\operatorname{USp}(4 n)$ down to its 'unextended' $\mathrm{U}(2 n)$ subgroup,

$$
\Psi \rightarrow S \Psi, \quad S=\left[\begin{array}{l|l}
U & \\
\hline & U^{*}
\end{array}\right], \quad U \in \mathrm{U}(2 n) .
$$

In the presence of both mass and chemical potential terms, the surviving global symmetry becomes $\mathrm{U}(n) \times$ $\mathrm{U}(n)$,

$$
\Psi \rightarrow S \Psi, \quad S=\left[\begin{array}{ll|ll}
u_{1} & & & \\
& u_{2} & & \\
\hline & & u_{1}^{*} & \\
& & u_{2}^{*}
\end{array}\right], \quad u_{1}, u_{2} \in \mathrm{U}(n),
$$

which is the intersection of $\operatorname{USp}(2 n) \times \operatorname{USp}(2 n)$ and $\mathrm{U}(2 n)$.

\section{Diquark source}

Due to the pseudoreality of the $\mathrm{SU}(2)$ gauge group, $\tau_{2} g \tau_{2}=g^{*}$, one can write down a gauge invariant bilinear of two quarks or two antiquarks. The source term for the $\left(\mathrm{P}, \mathbf{Z}_{2}\right)$-invariant diquark condensate

$$
\mathcal{L}_{j}=j\left(\psi^{T} \sigma_{2} \tau_{2} \chi+\bar{\psi} \sigma_{2} \tau_{2} \bar{\chi}^{T}\right)
$$

can thus be included (terms like $\psi^{T} \sigma_{2} \tau_{2} \psi$ vanishes by anticommutativity). It can be rewritten in terms of the $4 n$-flavored spinor,

$$
\mathcal{L}_{j}=\frac{j}{2} \Psi^{T} \sigma_{2} \tau_{2} \hat{J} \Psi, \hat{J}=\left[\begin{array}{ll|l}
\mathbb{1}_{n} & & \\
\hline & \mathbb{1}_{n} & \\
\hline & -\mathbb{1}_{n}
\end{array}\right] .
$$

This diquark source term and the mass term (15) belongs to the same multiplet under the $\operatorname{USp}(4 n)$ group, i.e. there exists a generator $X_{2}$ of $\operatorname{USp}(4 n)$ that rotates $\hat{M}$ into $\hat{J}$,

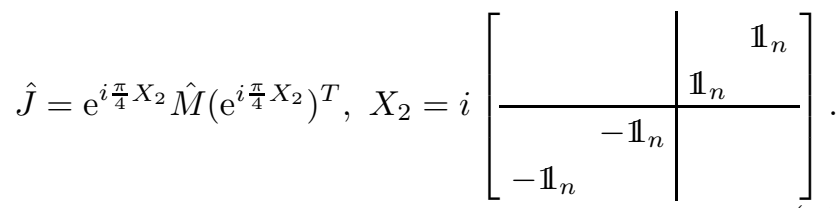

Namely the sum of mass and diquark source terms can be comprehensively written as

$$
\begin{aligned}
\mathcal{L}_{m}+\mathcal{L}_{j} & =\frac{1}{2} \Psi^{T} \sigma_{2} \tau_{2}(m \hat{M}+j \hat{J}) \Psi \\
& \equiv \frac{m \sec \phi}{2} \Psi^{T} \sigma_{2} \tau_{2} \hat{M}_{\phi} \Psi
\end{aligned}
$$

where we have defined the mixing angle $\phi$ by $\tan \phi=j / m$ and the rotated mass matrix $\hat{M}_{\phi}=\hat{M} \cos \phi+\hat{J} \sin \phi$. In constructing the effective theory in the following section, we shall set $j=0$. The effective Lagrangian can be translated to the case of nonvanishing $j$ by merely replacing $\hat{M} \rightarrow \hat{M}_{\phi}$ and $m \rightarrow m \sec \phi$. 


\section{LOW-ENERGY EFFECTIVE LAGRANGIAN}

\section{A. Global flavor symmetry}

We consider the effective theory resulting from the microscopic Lagrangian

$$
\begin{aligned}
\mathcal{L}= & -\frac{1}{2 g^{2}} \operatorname{tr} F_{\mu \nu} F_{\mu \nu}+\frac{1}{2} \Psi^{T} \sigma_{2} \tau_{2} \not D I \Psi \\
& +\frac{m}{2} \Psi^{T} \sigma_{2} \tau_{2} \hat{M} \Psi-\frac{\mu}{2} \Psi^{T} i \sigma_{1} \tau_{2} \hat{C} \Psi
\end{aligned}
$$

which is valid in the low energy $\left(\ll \Lambda_{\mathrm{QCD}}\right)$ regime where fundamental particles are confined and Goldstone bosons dominate. The kinetic term of the effective Lagrangian describing the Goldstone modes $\Sigma$, parametrized as in (17) and (18), should be invariant under the action of the global $\mathrm{USp}(4 n)$ group

$$
\Sigma(x) \rightarrow s \Sigma(x) s^{T}, \quad s \in \operatorname{USp}(4 n)
$$

with $s$ in the antisymmetric tensor representation. it should also be Lorentz invariant, and contain two derivatives. These requirements uniquely determine its form to be

$$
L_{\mathrm{kin}}=\frac{F^{2}}{2} \operatorname{tr} \partial_{\nu} \Sigma \partial_{\nu} \Sigma^{\dagger}
$$

up to a phenomenological "decay constant" $F$. This choice corresponds to normalizing the kinetic term as defined in (18), (23) to be the standard form $(1 / 2) \operatorname{tr} \partial_{\nu} \Pi \partial_{\nu} \Pi^{\dagger}$. The uniqueness is a consequence of the pseudo-reality of the field $\Sigma$,

$$
\Sigma^{\dagger}=I \Sigma I
$$

which follows from $\Sigma$ being symplectic, unitary and antisymmetric. One can systematically generate Skyrme-like higher derivative terms in the effective Lagrangian, but we do not pursue this issue in this paper as it is not necessary for our applications.

To identify the mass and chemical potential terms in the effective theory, one uses the well-established strategy of Gasser and Leutwyler 12. As the microscopic Lagrangian (32) can be made invariant under the global $\mathrm{USp}(4 n)$ transformation

$$
\Psi(x) \rightarrow s \Psi(x)
$$

by promoting the symmetry-breaking coupling constant matrices $m \hat{M}$ and $\mu \hat{C}$ to field variables that take these constant condensates in the vacuum and assigning the global transformation properties

$$
\hat{M}(x) \rightarrow s^{*} \hat{M}(x) s^{\dagger}, \quad \hat{C}(x) \rightarrow s^{*} \hat{C}(x) s^{\dagger},
$$

so should the low-energy effective Lagrangian be similarly invariant. The collection of local terms allowed by its invariance will then be reduced to the effective Lagrangian for $\Sigma(x)$ after the replacements $\hat{M}(x) \rightarrow \hat{M}$ and
$\hat{C}(x) \rightarrow \hat{C}$. To the lowest order in these couplings, the requirement of invariance determines the effective mass term to be

$$
L_{m}=-G m \operatorname{tr}(\hat{M} \Sigma),
$$

and the effective chemical potential term to be

$$
L_{\mu}=-H \mu^{2} \operatorname{tr}(\hat{C} \Sigma \hat{C} \Sigma)
$$

The first order term $\operatorname{tr}(\hat{C} \Sigma)$ trivially vanishes by symmetry. Other invariant combinations that appear seemingly different, such as $\operatorname{tr}\left(\hat{M}^{\dagger} \Sigma^{\dagger}\right)$, or $\operatorname{tr}\left(\hat{C}^{\dagger} \Sigma^{\dagger} \hat{C}^{\dagger} \Sigma^{\dagger}\right)$, $\operatorname{tr}\left(I \hat{C}^{\dagger} \Sigma^{\dagger} I \hat{C} \Sigma\right)$ and $\operatorname{tr}\left(\hat{C} I \Sigma^{\dagger} \hat{C}^{\dagger} I \Sigma\right)$, are equal to $\operatorname{tr}(\hat{M} \Sigma)$ or $\operatorname{tr}(\hat{C} \Sigma \hat{C} \Sigma)$, respectively, upon substitution of the vacuum condensates $\hat{M}(x) \rightarrow \hat{M}$ and $\hat{C}(x) \rightarrow \hat{C}$ and after some algebra using (35). Namely (38) and (39) are already real.

The coefficients $G$ and $H$ in (38) and (39) are phenomenological constants. Due to the relationship

$$
\langle\bar{\psi} \psi-\bar{\chi} \chi\rangle=-\frac{1}{V} \frac{\partial}{\partial m} Z(m, \mu)
$$

and the vacuum alignment of the field $\Sigma(x) \rightarrow \Sigma_{c}=\hat{M}^{\dagger}$ for $m \searrow 0, \mu \rightarrow 0$, [which will be shown below - see eq.(61)], the constant $G$ is equal to the quark-antiquark condensate at $m, \mu \rightarrow 0$,

$$
G=\left.\frac{1}{4 n} \lim _{m \searrow 0}\langle\bar{\psi} \psi-\bar{\chi} \chi\rangle\right|_{\mu=0} .
$$

\section{B. Local flavor symmetry}

Unlike the quark-antiquark condensate $G$, the phenomenological parameter $H$ is not independent of the decay constant $F$, but is related to it by virtue of the local flavor symmetry 16 . To lift the global USp $(4 n)$ symmetry to a local one, we combine $\mathcal{L}_{\text {kin }}$ and $\mathcal{L}_{\mu}$ into a covariant derivative form acting on the extended quark multiplet $\Psi$,

$$
\begin{aligned}
\mathcal{L}_{\text {kin }}+\mathcal{L}_{\mu} & =\frac{1}{2} \Psi^{T} \sigma_{2} \tau_{2} \sigma_{\nu} I\left(D_{\nu}-\mu B_{\nu}\right) \Psi \\
B_{\nu} & =\hat{B} \delta_{\nu, 3}, \quad \hat{B}=\hat{C} I=\left[\begin{array}{l|l|l}
\mathbb{1}_{2 n} & \\
\hline & -\mathbb{1}_{2 n}
\end{array}\right]
\end{aligned}
$$

This Lagrangian is invariant under the local $\operatorname{USp}(4 n)$ symmetry

$$
\Psi(x) \rightarrow s(x) \Psi(x), \quad s(x) \in \operatorname{USp}(4 n),
$$

when $B_{\nu}$ is promoted to a local field and is assigned the inhomogeneous transformation property of a flavor gauge field,

$$
B_{\nu}(x) \rightarrow s(x) B_{\nu}(x) s(x)^{\dagger}+\frac{1}{\mu}\left(\partial_{\nu} s(x)\right) s(x)^{\dagger},
$$


rather than the homogeneous global transformation (37). This local symmetry should also be respected by the effective Lagrangian, whose field variable transforms as

$$
\Sigma(x) \rightarrow s(x) \Sigma(x) s(x)^{T} .
$$

To cancel the extra two terms that appear in the transformation of the derivative of the field,

$$
\partial_{\nu} \Sigma \rightarrow s\left(\partial_{\nu} \Sigma\right) s^{T}+\left(\partial_{\nu} s\right) \Sigma s^{T}+s \Sigma\left(\partial_{\nu} s^{T}\right)
$$

one introduces flavor-covariant derivatives for the fields in the antisymmetric tensor representations

$$
\begin{aligned}
\nabla_{\nu} \Sigma & =\partial_{\nu} \Sigma-\mu\left(\Sigma B_{\nu}^{\dagger}+B_{\nu}^{\dagger} \Sigma\right) \\
\nabla_{\nu} \Sigma^{\dagger} & =\partial_{\nu} \Sigma^{\dagger}+\mu\left(\Sigma^{\dagger} B_{\nu}+B_{\nu} \Sigma^{\dagger}\right) .
\end{aligned}
$$

They transform covariantly

$$
\nabla_{\nu} \Sigma \rightarrow s\left(\nabla_{\nu} \Sigma\right) s^{T}, \quad \nabla_{\nu} \Sigma^{\dagger} \rightarrow s^{*}\left(\nabla_{\nu} \Sigma^{\dagger}\right) s^{\dagger},
$$

due to the gauge transformation property (44), while preserving the antisymmetry. Accordingly, the total effective Lagrangian reads

$$
\begin{aligned}
L & =L_{\mathrm{kin}}+L_{\mu}+L_{m} \\
& =\frac{F^{2}}{2} \operatorname{tr} \nabla_{\nu} \Sigma \nabla_{\nu} \Sigma^{\dagger}-G m \operatorname{tr}(\hat{M} \Sigma) \\
& =\frac{F^{2}}{2} \operatorname{tr} \partial_{\nu} \Sigma \partial_{\nu} \Sigma^{\dagger}+F^{2} \mu \operatorname{tr}\left(\left(\Sigma^{\dagger} \hat{B}+\hat{B} \Sigma^{\dagger}\right) \partial_{3} \Sigma\right) \\
& -F^{2} \mu^{2}\left(\operatorname{tr}\left(\hat{B} \Sigma \hat{B} \Sigma^{\dagger}\right)+4 n\right)-F^{2} m_{\pi}^{2} \operatorname{tr}(\hat{M} \Sigma)
\end{aligned}
$$

after partial integration on the linear derivative term. We observe that just as in [16], the parameter $H$ is not independent, but equal to $F^{2}$. In the last line of (49), we again used the pseudoreality of $\Sigma$ (35), and also replaced $G$ by the mass of the pseudo-Goldstone bosons $\pi_{a}(x)$ in (18) by the Gell-Mann-Oakes-Renner relation

$$
m_{\pi}^{2}=\frac{G m}{F^{2}}
$$

that results by expanding the fluctuation of the field $\Sigma(x)$ to second order (see the next section).

\section{VACUUM ALIGNMENT AND CONDENSATES}

The static part of the effective Lagrangian (49), i.e. the effective potential, determines the vacuum alignment of the field $\Sigma$. As in [16], we introduce a dimensionless phenomenological parameter $\xi=2 \mu / m_{\pi}$ to represent the chemical potential, and write

$$
L_{\mathrm{st}}(\Sigma)=F^{2} m_{\pi}^{2}\left(-\operatorname{tr}(\hat{M} \Sigma)-\frac{\xi^{2}}{4} \operatorname{tr}\left(\hat{B} \Sigma \hat{B} \Sigma^{\dagger}\right)-n \xi^{2}\right) .
$$

The above two terms compete for the direction of the condensate which we denote by $\bar{\Sigma}$.
To determine the vacuum condensate, we write $\Sigma$ in a block form (with each sub-block being $2 n \times 2 n=N_{F} \times$ $\left.N_{F}\right)$ :

$$
\Sigma=\left[\begin{array}{c|c}
\Sigma_{11} & \Sigma_{12} \\
\hline-\Sigma_{12}^{T} & \Sigma_{22}
\end{array}\right] .
$$

These component matrices also satisfy unitarity constraints

$$
\begin{aligned}
& \Sigma_{11} \Sigma_{11}^{\dagger}+\Sigma_{12} \Sigma_{12}^{\dagger}=\mathbb{1}_{2 n}, \\
& \Sigma_{12}^{T} \Sigma_{12}^{*}+\Sigma_{22} \Sigma_{22}^{\dagger}=\mathbb{1}_{2 n}, \\
& \Sigma_{11} \Sigma_{12}^{*}=\Sigma_{12} \Sigma_{22}^{\dagger},
\end{aligned}
$$

as well as constraints arising from the fact that $\Sigma$ is symplectic and antisymmetric. Using solely the unitarity constraints and the reality of $\operatorname{tr} \hat{M} \Sigma$, one can express $L_{\text {st }}$ entirely in terms of $\Sigma_{12}$,

$$
\begin{aligned}
& L_{\mathrm{st}}(\Sigma)= F^{2} m_{\pi}^{2}\left[\xi^{2} \operatorname{tr} \Sigma_{12} \Sigma_{12}^{\dagger}-\operatorname{tr} \Gamma \Sigma_{12}\right. \\
&\left.-\operatorname{tr}\left(\Gamma \Sigma_{12}\right)^{\dagger}-2 n \xi^{2}\right] \\
&=F^{2} m_{\pi}^{2}\left[\xi^{2} \operatorname{tr}\left(\Sigma_{12} \Gamma-\xi^{-2} \mathbb{1}_{2 n}\right)\left(\Sigma_{12} \Gamma-\xi^{-2} \mathbb{1}_{2 n}\right)^{\dagger}\right. \\
&\left.-2 n\left(\xi^{2}+\xi^{-2}\right)\right]
\end{aligned}
$$

where

$$
\Gamma=\left[\begin{array}{ll}
\mathbb{1}_{n} & \\
& -\mathbb{1}_{n}
\end{array}\right] .
$$

If we ignore the constraints on $\Sigma_{12}$ for a moment, the trace in (54) can be viewed from the distance between two points $\Sigma_{12}$ and $\xi^{-2} \mathbb{1}_{2 n}$ in the $2 n^{2}$-dimensional vector space of real and imaginary parts of the matrix elements. For $\xi>1$ the absolute minimum is achieved when $\Sigma_{12}=\xi^{-2} \Gamma$. The unitary, symplectic, and antisymmetry constraints on $\Sigma$ then determine the other sub-block matrices to be, up to the $\mathrm{U}(n)$ residual degree of freedom $u \in \mathrm{U}(n)$,

$$
\begin{aligned}
& \Sigma_{11}=\sqrt{1-\xi^{-4}}\left[\begin{array}{cr} 
& u \\
-u^{T} &
\end{array}\right], \\
& \Sigma_{22}=\sqrt{1-\xi^{-4}}\left[\begin{array}{c}
u^{*} \\
-u^{\dagger}
\end{array}\right] .
\end{aligned}
$$

When $\xi<1$, the unitarity constraints (53) demand that

$$
\operatorname{tr}\left(\Sigma_{12} \Gamma\right)\left(\Sigma_{12} \Gamma\right)^{\dagger} \leq 2 n,
$$

and the point $\xi^{-2} \mathbb{1}_{2 n}$ lies outside of this region. The point closest to $\xi^{-2} \mathbb{1}_{2 n}$ within this $2 n^{2}$-dimensional ball is clearly at $\Sigma_{12} \Gamma=\mathbb{1}_{2 n}$. Then the unitarity constraints (53) can be satisfied only by $\Sigma_{11}=\Sigma_{22}=0$. To summarize, the condensate that gives the global minimum of 
the static effective Lagrangian is

$$
\bar{\Sigma}=\hat{M}^{\dagger} \cos \alpha+\left[\begin{array}{l|l}
-u^{T} & \\
\hline & -u^{\dagger}
\end{array}\right] \sin \alpha
$$

where $u \in \mathrm{U}(n)$, and we have defined

$$
\cos \alpha=\min \left(1, \xi^{-2}\right) .
$$

Note that the first term of the condensate (58) does not carry baryon number, while the second term does.

The condensate is a non-analytic function of $\xi=$ $2 \mu / m$. In the regime $\xi>1$, the vacuum condensate has the $\mathrm{U}(n)$ degeneracy corresponding to $n^{2}$ true Goldstone modes. This change of massless modes indicates a second-order phase transition at $\xi=1$. The static effective Lagrangian is

$$
\begin{aligned}
L_{\text {st }}(\bar{\Sigma}) & =-4 n F^{2} m_{\pi}^{2} \times \begin{cases}1 & (\xi<1) \\
\left(\xi^{2}+\xi^{-2}\right) / 2 & (\xi>1)\end{cases} \\
& = \begin{cases}-4 n G m & \left(\mu<m_{\pi} / 2\right) \\
-n\left(8 F^{2} \mu^{2}+\frac{G^{2} m^{2}}{2 F^{2} \mu^{2}}\right) & \left(\mu>m_{\pi} / 2\right)\end{cases}
\end{aligned}
$$

The second $\xi$-derivative of the $L_{\text {st }}$ is indeed discontinuous at $\xi=1$.

The quark-antiquark condensate $\langle\bar{\psi} \psi-\bar{\chi} \chi\rangle$ and the baryon density $\left\langle\psi^{\dagger} \psi+\chi^{\dagger} \chi\right\rangle$ directly follow from (60). Upon differentiation with respect to $m$ and $\mu$, respectively, we find

$$
\begin{aligned}
\langle\bar{\psi} \psi-\bar{\chi} \chi\rangle & = \begin{cases}4 n G & (\xi<1) \\
4 n G \xi^{-2} & (\xi>1)\end{cases} \\
\left\langle\psi^{\dagger} \psi+\chi^{\dagger} \chi\right\rangle & = \begin{cases}0 & (\xi<1) \\
16 n F^{2} \mu\left(1-\xi^{-4}\right) & (\xi>1)\end{cases}
\end{aligned}
$$

These condensates are identical (with the replacement $2 n \rightarrow N_{f}$ ) to the corresponding condensates in the 4D $S U(2)$ case, with fundamental quarks, studied in [17] see Table 3 in [17]. This follows from the fact that the vacuum energy has the same functional form in the $3 \mathrm{D}$ and $4 \mathrm{D}$ cases. This is nontrivial from the microscopic point of view since the symmetries involved are completely different. Rather, this universality arises from the mean field treatment of the low-energy effective theory, as we discuss further in the conclusions.

\section{CURVATURE AT THE MINIMUM}

In this section we expand the static effective Lagrangian around the vacuum condensate (58) to second order. In the diquark condensation phase $\xi>1$, we arbitrarily fix the $\mathrm{U}(n)$ degeneracy by $u=\mathbb{1}_{n}$,

$$
\bar{\Sigma}_{\alpha}=\hat{M}^{\dagger} \cos \alpha+\hat{J} \sin \alpha
$$

with $\alpha$ given by (59). At small chemical potential in the range $0 \leq \xi<1(\alpha=0)$ this condensate aligns to $\hat{M}^{\dagger}$, which is preserved by the $\operatorname{USp}(2 n) \times \operatorname{USp}(2 n)$ subgroup acting on the $(1,3)$ and $(2,4)$ blocks : $\Sigma \rightarrow S \Sigma S^{T}$, with $S$ given by (16). Namely for $0<\xi<1$ the mass term explicitly breaks this symmetry down to $\mathrm{U}(n) \times \mathrm{U}(n)$ given by (27), also acting on the $(1,3)$ and $(2,4)$ blocks. On the other hand, in the massless limit $\xi=\infty$ this condensate aligns to $\hat{J}$, and the residual global symmetry becomes the $\operatorname{USp}(2 n)$ subgroup,

$$
\Sigma \rightarrow\left[\begin{array}{l|l}
s & \\
\hline & s^{*}
\end{array}\right] \Sigma\left[\begin{array}{l|l}
s^{T} & \\
\hline & s^{\dagger}
\end{array}\right], \quad s \in \operatorname{USp}(2 n),
$$

of the flavor $\operatorname{USp}(4 n)$ group. In the intermediate region $1<\xi<\infty$ the residual symmetry is the $\mathrm{U}(n)$ subgroup given by (27) with $u_{2}=u_{1}^{*}$. All excitations will be classified according to the representations of these residual symmetry groups.

\section{A. Normal phase}

When $\xi<1$ the vacuum orientation of the condensate does not depend on $\xi$ and is given by $\bar{\Sigma}=\hat{M}^{\dagger}$. Expanding $\Sigma$ around $\hat{M}^{\dagger}$ using the Goldstone field defined in (18) according to

$$
\Sigma=S \hat{M}^{\dagger} S^{T}=S^{2} \hat{M}^{\dagger}=\left(1+\frac{i \Pi}{F}-\frac{\Pi^{2}}{2 F^{2}}+\cdots\right) \hat{M}^{\dagger},
$$

we find

$$
L_{\mathrm{st}}(\Sigma)=L_{\mathrm{st}}\left(\bar{\Sigma}_{0}\right)+\frac{m_{\pi}^{2}}{2}\left[\operatorname{tr} \Pi^{2}+\frac{\xi^{2}}{4} \operatorname{tr}[\hat{B}, \Pi]^{2}\right]+\cdots,
$$

where the ellipsis denotes terms of higher order in $\Pi$. Substituting the parametrization (23), we obtain

$$
\begin{aligned}
L_{\mathrm{st}}(\Sigma) & =L_{\mathrm{st}}\left(\bar{\Sigma}_{0}\right) \\
& +\frac{m_{\pi}^{2}}{2}\left[\operatorname{tr} P P^{\dagger}+\left(1-\xi^{2}\right) \operatorname{tr} Q Q^{\dagger}\right]+\cdots, \\
L_{\mathrm{st}}\left(\bar{\Sigma}_{0}\right) & =-4 n F^{2} m_{\pi}^{2} .
\end{aligned}
$$

This confirms that the configuration (63) is indeed a minimum. We also see that there are no true Goldstone modes for $\xi<1$. At $\xi=1$ the curvature of the diquark modes $Q$ vanish, which signals a second order phase transition and the diquark condensation.

Below we list the representations of the residual symmetry group $\mathrm{U}(n) \times \mathrm{U}(n)$ to which the fields belong by listing the representations' Young tableaux,

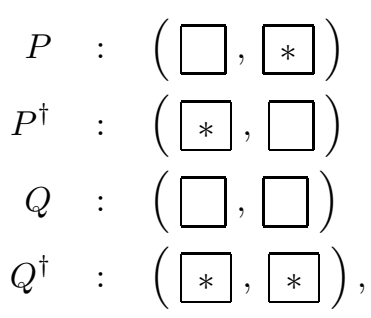


where $\square$ and $*$ stand for fundamental and conjugated fundamental representations, respectively. The dimensions of these representations are all equal to $n^{2}$. At vanishing chemical potential, $\xi=0$, these four fields combine to become a $4 n^{2}$-dimensional multiplet $(\square, \square)$ of $\mathrm{USp}(2 n) \times \operatorname{USp}(2 n)$.

\section{B. Diquark condensation phase}

When $\xi>1$ the configuration (63) begins to rotate according to $\cos \alpha=\xi^{-2}$. This rotation can also be written as

$$
\bar{\Sigma}_{\alpha}=s_{\alpha} \hat{M}^{\dagger} s_{\alpha}^{T}=s_{\alpha}^{2} \hat{M}^{\dagger}, \quad s_{\alpha}=\mathrm{e}^{i \frac{\alpha}{2} X_{2}},
$$

where $X_{2}$ is the generator, defined in (30), that rotates $\hat{M}$ into $\hat{J}$. We could parametrize the fluctuation around the vacuum $\bar{\Sigma}_{\alpha}$ as

$$
\Sigma=S_{\alpha} \bar{\Sigma}_{\alpha} S_{\alpha}^{T}=S_{\alpha} s_{\alpha} \hat{M}^{\dagger} s_{\alpha}^{T} S_{\alpha}^{T},
$$

where $S_{\alpha}$ are symplectic matrices generated by rotated generator $s_{\alpha} X_{a} s_{\alpha}^{\dagger}$, instead of $X_{a}$. However, we employ an alternative parametrization of the fluctuation,

$$
\Sigma=s_{\alpha} S \hat{M}^{\dagger} S^{T} s_{\alpha}^{T}
$$

where $S$ are generated by unrotated generators $X_{a}$, in order for the meson mass matrix to be diagonal subsequently. We substitute this parametrization into the static effective Lagrangian (51) and obtain

$$
\begin{aligned}
& L_{\mathrm{st}}(\Sigma)=F^{2} m_{\pi}^{2}\left[-\operatorname{tr}\left(\hat{M}^{\dagger} s_{\alpha}^{T} \hat{M} s_{\alpha} S^{2}\right)\right. \\
& \left.-\frac{\xi^{2}}{4} \operatorname{tr}\left(\hat{M}^{\dagger} s_{\alpha}^{T} \hat{B} s_{\alpha}^{*} \hat{M}\left(S^{\dagger}\right)^{2} s_{\alpha}^{\dagger} \hat{B} s_{\alpha} S^{2}\right)-n \xi^{2}\right] .
\end{aligned}
$$

Substituting the parametrizations (18) and (23) into (73), and expanding to second order in $P$ and $Q$, we find

$$
\begin{aligned}
L_{\mathrm{st}}(\Sigma)= & L_{\mathrm{st}}\left(\bar{\Sigma}_{\alpha}\right)+\frac{m_{\pi}^{2}}{2}\left[\xi^{2} \operatorname{tr} P_{S} P_{S}^{\dagger}+\xi^{-2} \operatorname{tr} P_{A} P_{A}^{\dagger}\right. \\
& \left.+\left(\xi^{2}-\xi^{-2}\right) \operatorname{tr} Q_{I}^{2}\right]+\cdots \\
L_{\mathrm{st}}\left(\bar{\Sigma}_{\alpha}\right)= & -2 n\left(\xi^{2}+\xi^{-2}\right) F^{2} m_{\pi}^{2}
\end{aligned}
$$

Here we have defined $P_{S}, P_{A}, Q_{R}, Q_{I}$ as

$$
\begin{aligned}
& P_{S}=\frac{P+P^{T}}{2}=P_{S}^{T} \quad: \quad \square \quad \square, \begin{array}{|l|l|}
\hline * & * \\
\hline
\end{array} \\
& P_{A}=\frac{P-P^{T}}{2}=-P_{A}^{T}: \square, \quad \square \\
& Q_{R}=\frac{Q+Q^{\dagger}}{2}=Q_{R}^{\dagger} \quad: \quad * \quad \\
& Q_{I}=\frac{Q-Q^{\dagger}}{2 i}=Q_{I}^{\dagger} . \quad: \quad * \quad
\end{aligned}
$$

These projections are orthogonal, $\operatorname{tr} P_{S} P_{A}=$ $\Im m \operatorname{tr} Q_{R} Q_{I}=0$. The representations of the residual symmetry group $\mathrm{U}(n)$ to which the fields belong are denoted by the Young tableaux. The dimensions of these representations are $n^{2}+n, n^{2}-n, n^{2}$ and $n^{2}$, respectively.

From (74) we can read off the curvatures of the different multiplets of the pseudo-Goldstone modes, and again confirm the local minimality of the configuration (63). We see that there are $n^{2}$ true flat directions, $Q_{R}$, which describe massless Goldstone modes. By contrasting this fact with the solution (58) at $\xi \rightarrow \infty$, the true Goldstone fields are identified with the $\mathrm{U}(n)$ phases of the diquark condensate. As we shall see in the next section, two modes $Q_{R}$ and $Q_{I}$ that belong to the same representation are mixed by the linear derivative terms in the effective Lagrangian, and thus the actual true Goldstone excitations are certain linear combinations of $Q_{R}$ and $Q_{I}$.

\section{MASS SPECTRUM}

In order to determine the spectrum of low-lying excitations we must take into account the derivative terms in the effective Lagrangian (49). The dispersion laws of the system at finite chemical potential do not take the simple Lorenz invariant form $E^{2}=\mathbf{p}^{2}+M^{2}$. We shall evaluate the pole mass (rest energy) that is the value $E=i p_{3}$ of the pole of the propagator at $\mathbf{p}=0$.

\section{A. Normal phase}

Expanding the derivative terms in the effective Lagrangian (49) in the same way as we expanded the potential part in the previous section, we obtain

$$
\begin{aligned}
L & =L_{\mathrm{st}}\left(\bar{\Sigma}_{\alpha}\right)+\frac{1}{2} \operatorname{tr} \partial_{\nu} P^{\dagger} \partial_{\nu} P+\frac{m_{\pi}^{2}}{2} \operatorname{tr} P^{\dagger} P \\
& +\frac{1}{2} \operatorname{tr} \partial_{\nu} Q^{\dagger} \partial_{\nu} Q-2 \mu \operatorname{tr} Q^{\dagger} \partial_{3} Q+\left(\frac{m_{\pi}^{2}}{2}-2 \mu^{2}\right) \operatorname{tr} Q^{\dagger} Q \\
& +\cdots
\end{aligned}
$$

After using the Fourier decomposition of $\Pi$,

$$
\Pi(x)=\sum_{p} \Pi_{p} \mathrm{e}^{-i p x}, \quad p=(\mathbf{p},-i E)
$$

the equations of motion for $P, P^{\dagger}, Q, Q^{\dagger}$ that follow from (177) lead to the dispersion law

$$
(E+b \mu)^{2}=\mathbf{p}^{2}+m_{\pi}^{2} .
$$

Here $b$ is the baryon charge of the corresponding excitation, assigned as : $b=0$ for $P$ and $P^{\dagger} ; b=2$ for $Q$; and $b=-2$ for $Q^{\dagger}$. Accordingly, the pole masses for these excitations are

$$
\begin{aligned}
m_{\pi} \text { for } P \text { and } P^{\dagger} \\
m_{\pi}-2 \mu \text { for } Q \\
m_{\pi}+2 \mu \text { for } Q^{\dagger}
\end{aligned}
$$




\section{B. Diquark condensation phase}

In this phase the ground state changes and we need to expand around the rotated value of the condensate $\bar{\Sigma}_{\alpha}$, as in (72). To second order in the fluctuation $\Pi$ we obtain

$$
\begin{aligned}
L & =L_{\mathrm{st}}\left(\bar{\Sigma}_{0}\right)+\frac{1}{2} \operatorname{tr} \partial_{\nu} P_{S}^{\dagger} \partial_{\nu} P_{S}+\frac{1}{2} \operatorname{tr} \partial_{\nu} P_{A}^{\dagger} \partial_{\nu} P_{A} \\
& +\frac{1}{2} \operatorname{tr}\left(\partial_{\nu} Q_{R}\right)^{2}+\frac{1}{2} \operatorname{tr}\left(\partial_{\nu} Q_{I}\right)^{2} \\
& +2 i \mu \xi^{-2} \operatorname{tr}\left(Q_{R}^{\dagger} \partial_{3} Q_{I}-Q_{I}^{\dagger} \partial_{3} Q_{R}\right) \\
& +\frac{m_{\pi}^{2}}{2}\left[\xi^{2} \operatorname{tr} P_{S} P_{S}^{\dagger}+\xi^{-2} \operatorname{tr} P_{A} P_{A}^{\dagger}+\left(\xi^{2}-\xi^{-2}\right) \operatorname{tr} Q_{I}^{2}\right] \\
& +\cdots
\end{aligned}
$$

The linear derivative term contains only the $Q$ fields, which have a nonzero baryon charge, and the dispersion law for the $P$ fields remain Lorentz invariant in form:

$$
\begin{aligned}
& P_{S}: \quad E^{2}=\mathbf{p}^{2}+m_{\pi}^{2} \xi^{2} \\
& P_{A}: \quad E^{2}=\mathbf{p}^{2}+m_{\pi}^{2} \xi^{-2} .
\end{aligned}
$$

On the other hand, the dispersion laws for the $Q$ fields are determined by the secular equation obtained by substitution of the Fourier decomposition of $Q_{R}$ and $Q_{I}$,

$$
\operatorname{det}\left[\begin{array}{cc}
{\left[E^{2}-\mathbf{p}^{2}\right]} & 4 i E \mu \xi^{-2} \\
-4 i E \mu \xi^{-2} & {\left[E^{2}-\mathbf{p}^{2}-m_{\pi}^{2}\left(\xi^{2}-\xi^{-2}\right)\right]}
\end{array}\right]=0 .
$$

We see that $Q_{R}$ and $Q_{I}$ are mixed by the linear derivative term unless $\cos \alpha=0$, i.e. in the massless limit. Due to the form of (83), for any $\alpha$ there is a solution for which $E(\mathrm{p}=0)=0$, and the true Goldstone mode is denoted by $\tilde{Q}$. In the massless limit it is entirely $Q_{R}$. The other massive solution of the secular equation, which is a linear combination of $Q_{R}$ and $Q_{I}$ orthogonal to $\tilde{Q}$, is denoted by $\tilde{Q}^{\dagger}$. The dispersion laws for these fields are given by

$$
\begin{aligned}
\tilde{Q}: E^{2} & =\mathbf{p}^{2}+2 \mu^{2}\left(1+3 \xi^{-4}\right) \\
& -2 \mu \sqrt{\mu^{2}\left(1+3 \xi^{-4}\right)^{2}+4 \mathbf{p}^{2} \xi^{-2}}, \\
\tilde{Q}^{\dagger}: E^{2} & =\mathbf{p}^{2}+2 \mu^{2}\left(1+3 \xi^{-4}\right) \\
& +2 \mu \sqrt{\mu^{2}\left(1+3 \xi^{-4}\right)^{2}+4 \mathbf{p}^{2} \xi^{-2}} .
\end{aligned}
$$

The pole masses of these four excitations, i.e. $E(\mathrm{p}=0)$, are then given by

$$
\begin{array}{r}
2 \mu \text { for } P_{S} \\
\frac{m_{\pi}^{2}}{2 \mu} \text { for } P_{A} \\
0 \text { for } \tilde{Q} \\
2 \mu \sqrt{1+3\left(\frac{m_{\pi}}{2 \mu}\right)^{4}} \text { for } \tilde{Q}^{\dagger}
\end{array}
$$

These dispersion laws and pole masses are identical to their 4D counterparts: see Eq.(86) of KSTVZ. This is a remarkable manifestation of the universality of the low-energy effective theories governing quasi-Goldstone bosons.

We finally note that in the massless limit $P_{S}$ and $\tilde{Q}^{\dagger}=Q_{I}$ combine to become a $\left(2 n^{2}+n\right)$-dimensional multiplet $\square$ of $\operatorname{USp}(2 n)$, and $P_{A}$ and $\tilde{Q}=Q_{R}$ combine to become a $\left(2 n^{2}-n\right)$-dimensional multiplet $\square$ of $\mathrm{USp}(2 n)$.

\section{CONCLUSION}

In this paper we have used the effective field theory method at finite baryon density to investigate the phase structure and the excitation spectrum of the three dimensional parity invariant SU(2) QCD with fundamental quarks. Since the quarks are in a pseudoreal representation we can introduce a real chemical potential for baryon number, as was done for 4D in 16, 17]. Our main result is that even though the symmetry breaking groups are different in $3 \mathrm{D}$ and $4 \mathrm{D}$, the final phase structure, condensates and dispersion relations are the same in $3 \mathrm{D}$ and 4D. Furthermore, it is straightforward to generalize this to the case with adjoint fermions (and any $\mathrm{SU}\left(N_{C}\right)$ gauge group); at the level of fundamental Lagrangian one merely needs to ignore the color $\tau_{2}$ matrices altogether. This leads to replacing the USp groups that appear in the global symmetry arguments by the $\mathrm{O}$ groups of the same dimension due to the change of the constant matrices,

$$
\begin{aligned}
& I=-\left[\begin{array}{l|l} 
& \mathbb{1}_{2 n} \\
\hline \mathbb{1}_{2 n} &
\end{array}\right], \hat{M}=\left[\begin{array}{ll|ll} 
& & -\mathbb{1}_{n} & \\
& & \mathbb{1}_{n} \\
\hline-\mathbb{1}_{n} & & & \\
& \mathbb{1}_{n} & &
\end{array}\right],
\end{aligned}
$$

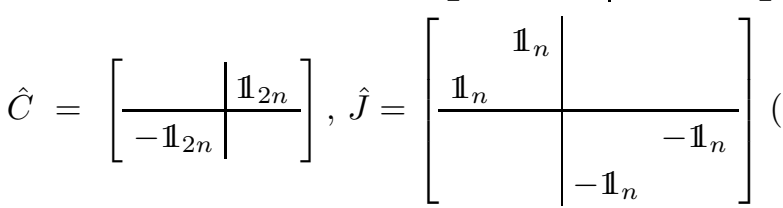

(the matrix $\hat{B}=\hat{C} I$ is left unchanged from (42) except for an irrelevant flip of the sign), and accordingly to interchanging the symmetric and antisymmetric properties of the field $\Sigma(x)$ and the representations of various excitations. Thus we have shown that the low-energy behavior of (pseudo-)real gauge theories at finite baryon density is universal beyond the dimensionality of spacetime and the representation of the quarks. The individuality of each case resides solely in the number of modes belonging to a multiplet with common mass.

To compute the diquark condensate $\left\langle\psi^{T} \sigma_{2} \tau_{2} \chi-\psi^{\dagger} \sigma_{2} \tau_{2} \chi^{*}\right\rangle$, one should include its source term in the effective Lagrangian by replacing the matrix $\hat{M}$ by $\hat{M}_{\phi}$ and $m$ by $m \sec \phi$, as mentioned at the end of sect[ID This modification of the mass term smears the abrupt change of the chemical potential 
dependence of the vacuum condensate at $\xi=1$, and turns the second-order phase transition into a crossover, just as in the $4 \mathrm{D}$ case [17. This is a straightforward exercise and we do not record the details here. The final results for the chemical potential dependences of the quark-antiquark condensate, diquark condensate, and baryon density as well as the dispersion laws for the excitations are identical to the $4 \mathrm{D}$ case.

The universality becomes most transparent by expressing the whole argument in terms of the generic symmetric spaces [30] that arise when a global symmetry group is broken. Let $G$ be a compact Lie group and $\mathcal{G}$ its Lie algebra $G=\exp \mathcal{G}$. For $G$ to be identified as a global symmetry of a gauge theory with (pseudo-)real quarks, its dimension is set to be even. An involutive automorphism $\sigma$ of $\mathcal{G}$ is defined as

$$
\sigma: \mathcal{G} \rightarrow \mathcal{G}, X \mapsto \sigma(X), \sigma^{2}=1 .
$$

An involution $\sigma$ naturally introduces a decomposition (known as the Cartan decomposition) of $\mathcal{G}$ with respect to $\sigma$. This decomposition splits $\mathcal{G}$ into a subalgebra with eigenvalue +1 , and a subspace orthogonal to it with eigenvalue -1 ,

$$
\mathcal{G}=\mathcal{K} \oplus \mathcal{P}, \quad \sigma(X)= \pm X \text { if } X \in\left\{\begin{array}{l}
\mathcal{K} \\
\mathcal{P}
\end{array} .\right.
$$

Below we will identify certain choices for this involution with mass and chemical potential terms.

Denote by $K$ the Lie subgroup generated by $\mathcal{K}, K=$ $\exp \mathcal{K}$. The Riemannian symmetric space $G / K$ is isomorphic to the exponential map of $\mathcal{P}, G / K \simeq \exp \mathcal{P}$ through the Cartan mapping $\mathcal{C}$,

$$
\mathcal{C}(g)=g \sigma(g)^{-1} .
$$

Now we choose an involution of the form

$$
\sigma(g)=\hat{M}^{-1} g^{*} \hat{M}, \quad \hat{M}^{*} \hat{M}= \pm 1, \quad \hat{M}^{\dagger} \hat{M}=1,
$$

which introduces a Cartan decomposition $\mathcal{G}=\mathcal{K} \oplus \mathcal{P}$, and specifies the unbroken vector subgroup $K$, determined by the dynamics. This involution will be associated with the mass term. The low energy effective theory for the Goldstone modes is a nonlinear $\sigma$ model on $G / K$; that is, it is a field theory whose field variable is parametrized by $\mathcal{C}(g)$ for $g \in G$. The potential of the nonlinear sigma model should also be invariant under the left action of $K$ on $G, g \mapsto k g$, which acts adjointly on $\mathcal{C}(g)$ by $\mathcal{C}(g) \mapsto k \mathcal{C}(g) k^{-1}$. Such an invariant quantity is spanned by $\left\{\operatorname{tr} \mathcal{C}(g)^{\nu}\right\}_{\nu \in \mathbf{Z}}$. We define the mass term in the effective field theory to be the combination that is real and of lowest order, i.e. $\Re \operatorname{tr} \mathcal{C}(g)$.

Next we fix another involution of the form

$$
\tilde{\sigma}(g)=\hat{B}^{-1} g \hat{B}, \quad \hat{B}^{2}=1 .
$$

which introduces another Cartan decomposition $\mathcal{G}=$ $\tilde{\mathcal{K}} \oplus \tilde{\mathcal{P}}$, with associated Cartan mapping $\tilde{\mathcal{C}}(g)=g \tilde{\sigma}(g)^{-1}$. For this involution $\tilde{\sigma}$ to be identified with the baryon charge, we take $\hat{B}=\operatorname{diag}(1, \ldots, 1,-1, \ldots,-1)$. The corresponding symmetry breaking term in the effective Lagrangian must be expressed in terms of the field variable $\mathcal{C}(g)$, and must be invariant only under the left action of $K \cap \tilde{K}$ on $G$. These terms are spanned by $\left\{\operatorname{tr} \tilde{\mathcal{C}}\left(\mathcal{C}(g)^{\nu}\right)^{\lambda}\right\}_{\nu, \lambda \in \mathbf{Z}}$. We define the chemical potential term to be the combination that is real and of lowest nontrivial order, i.e. $\operatorname{tr} \tilde{\mathcal{C}}(\mathcal{C}(g))$.

Thus, the static part of the effective Lagrangian is

$$
L_{\mathrm{st}}=-\Re \mathrm{e} \operatorname{tr} \mathcal{C}(g)+\frac{\xi^{2}}{4} \operatorname{tr} \tilde{\mathcal{C}}(\mathcal{C}(g))
$$

In order to find the extremum of $L_{\mathrm{st}}$, we decompose $\mathcal{C}(g)$ into four blocks according to those of $\hat{B}$,

$$
\mathcal{C}(g)=\left[\begin{array}{l|l}
\mathcal{C}_{11} & \mathcal{C}_{12} \\
\hline \mathcal{C}_{21} & \mathcal{C}_{22}
\end{array}\right] .
$$

Using the unitarity constraints on $\mathcal{C}_{i j}, L_{\text {st }}$ now reads

$$
\begin{aligned}
L_{\mathrm{st}} & =\frac{\xi^{2}}{2} \operatorname{tr}\left(\mathcal{C}_{11}-\xi^{-2}\right)\left(\mathcal{C}_{11}-\xi^{-2}\right)^{\dagger} \\
& +\frac{\xi^{2}}{2} \operatorname{tr}\left(\mathcal{C}_{22}-\xi^{-2}\right)\left(\mathcal{C}_{22}-\xi^{-2}\right)^{\dagger}+\text { const. }
\end{aligned}
$$

This factorized form covers the various $4 \mathrm{D}$ cases treated in KSTVZ [17], as well as the 3D cases considered in this paper. The important point for the universality of the form of this static effective Lagrangian is that one does not need to specify whether the original global symmetry group is $\mathrm{SU}\left(2 N_{F}\right)$ or $\mathrm{USp}\left(2 N_{F}\right)$ or $\mathrm{O}\left(2 N_{F}\right)$. The two-phase structure is manifest from this form of $L_{\mathrm{st}}$, as shown in [17] and in section IV of this paper.

The universality of the mass spectrum can be understood similarly : the kinetic term of the nonlinear $\sigma$ model is naturally derived from the metric on $G / K$ that is induced from the Killing form on $G$. We can choose a $\mathcal{G}$-valued connection $B_{\mu}$ on the $G$-bundle over the spacetime manifold and construct an associated LaplaceBeltrami operator $\nabla^{2}$. This procedure leads directly to the universality of the excitation masses.

\section{Acknowledgments}

We thank Alex Kovner for participating in the early stages of this work, and Jac Verbaarschot for helpful discussions and comments. We also thank the U.S. DOE for support through the grant DE-FG02-92ER40716. 
[1] V.A. Miransky, "Dynamical Symmetry Breaking in Quantum Field Theories", World Scientific (Singapore, 1993).

[2] C. Vafa and E. Witten, "Restrictions on symmetry breaking in vector-like gauge theories," Nucl. Phys. B 234, 173 (1984); "Eigenvalue inequalities for fermions in gauge theories," Commun. Math. Phys. 95, 257 (1984); "Parity conservation in QCD," Phys. Rev. Lett. 53, 535 (1984).

[3] R. Jackiw and S. Templeton, "How superrenormalizable interactions cure their infrared divergences," Phys. Rev. D 23, 2291 (1981).

[4] A. P. Polychronakos, "Symmetry breaking patterns in (2+1)-dimensional gauge theories," Phys. Rev. Lett. 60, 1920 (1988).

[5] R. D. Pisarski, "Chiral symmetry breaking in threedimensional electrodynamics," Phys. Rev. D 29, 2423 (1984).

[6] K. Stam, "Dynamical mass generation in large N QED in three-dimensions," Phys. Rev. D 34, 2517 (1986).

[7] T. Appelquist, M. J. Bowick, D. Karabali and L. C. Wijewardhana, "Spontaneous breaking of parity in $(2+1)$ dimensional QED," Phys. Rev. D 33, 3774 (1986); T. W. Appelquist, M. J. Bowick, D. Karabali and L. C. Wijewardhana, "Spontaneous chiral symmetry breaking in three-dimensional QED," Phys. Rev. D 33, 3704 (1986); T. Appelquist, D. Nash and L. C. Wijewardhana, "Critical behavior in (2+1)-dimensional QED," Phys. Rev. Lett. 60, 2575 (1988); D. Nash, "Higher order corrections in (2+1)-dimensional QED," Phys. Rev. Lett. 62, 3024 (1989); T. Appelquist, J. Terning and L. C. Wijewardhana, "(2+1)-dimensional QED and a novel phase transition," Phys. Rev. Lett. 75, 2081 (1995) arXiv:hep-ph/9402320.

[8] M. C. Diamantini, P. Sodano and G. W. Semenoff, "Chiral dynamics and fermion mass generation in threedimensional gauge theory," Phys. Rev. Lett. 70, 3848 (1993) arXiv:hep-ph/9301256.

[20] E. Dagotto, J. B. Kogut and A. Kocic, "A computer simulation of chiral symmetry breaking in $(2+1)$-dimensional QED with N flavors," Phys. Rev. Lett. 62, 1083 (1989); "Chiral symmetry breaking in three-dimensional QED with N(F) flavors," Nucl. Phys. B 334, 279 (1990).

[10] M. R. Pennington and D. Walsh, "Masses from nothing: A nonperturbative study of QED in three-dimensions," Phys. Lett. B 253, 246 (1991); D. C. Curtis, M. R. Pennington and D. Walsh, "Dynamical mass generation in QED in three-dimensions and the $1 / \mathrm{N}$ expansion," Phys. Lett. B 295, 313 (1992).

[11] R. D. Pisarski, "Fermion mass in three-dimensions and the renormalization group," Phys. Rev. D 44, 1866 (1991).

[12] J. Gasser and H. Leutwyler, "Chiral perturbation theory to one loop," Annals Phys. 158, 142 (1984); "Chiral perturbation theory: Expansions in the mass of the strange quark," Nucl. Phys. B 250, 465 (1985).

[13] S. Weinberg, "The Quantum Theory of Fields, Vol. 2: Modern Applications," sect.19, Cambridge Univ. Press (Cambridge, UK, 1996).
[14] J. J. M. Verbaarschot and T. Wettig, "Random matrix theory and chiral symmetry in QCD," Ann. Rev. Nucl. Part. Sci. 50, 343 (2000) arXiv:hep-ph/0003017.

[15] S. Hands, J. B. Kogut, M. P. Lombardo and S. E. Morrison, "Symmetries and spectrum of $\mathrm{SU}(2)$ lattice gauge theory at finite chemical potential," Nucl. Phys. B 558, 327 (1999) arXiv:hep-lat/9902034.

[16] J. B. Kogut, M. A. Stephanov and D. Toublan, "On twocolor QCD with baryon chemical potential," Phys. Lett. B 464, 183 (1999) arXiv:hep-ph/9906346.

[17] J. B. Kogut, M. A. Stephanov, D. Toublan, J. J. M. Verbaarschot and A. Zhitnitsky, "QCD-like theories at finite baryon density," Nucl. Phys. B 582, 477 (2000) arXiv:hep-ph/0001171.

[18] D. Toublan and J. J. M. Verbaarschot, "Dirac spectra and real QCD at nonzero chemical potential," arXiv:hep-th/0208021

[19] J. J. M. Verbaarschot, "What really matters", talk at the Channel Meeting, Plymouth, August 2002.

[20] E. Dagotto, F. Karsch and A. Moreo, "The strong coupling limit of SU(2) QCD at finite baryon density," Phys. Lett. B 169, 421 (1986).

[21] C. Baillie, K. C. Bowler, P. E. Gibbs, I. M. Barbour and M. Rafique, "The chiral condensate in SU(2) QCD at finite density," Phys. Lett. B 197, 195 (1987).

[22] J. B. Kogut, D. Toublan and D. K. Sinclair, "The phase diagram of four flavor $\mathrm{SU}(2)$ lattice gauge theory at nonzero chemical potential and temperature," Nucl. Phys. B 642, 181 (2002) arXiv:hep-lat/0205019; "SU(2) lattice gauge theory at nonzero chemical potential and temperature," arXiv:hep-lat/0208076

[23] P. H. Damgaard, U. M. Heller, A. Krasnitz and T. Madsen, "A quark-antiquark condensate in threedimensional QCD," Phys. Lett. B 440, 129 (1998) arXiv:hep-lat/9803012.

[24] S. R. Coleman and E. Witten, "Chiral symmetry breakdown In large N chromodynamics," Phys. Rev. Lett. 45, 100 (1980).

[25] U. Magnea, "The orthogonal ensemble of random matrices and QCD in three dimensions," Phys. Rev. D 61, 056005 (2000) arXiv:hep-th/9907096.

[26] M. E. Peskin, "The alignment of the vacuum in theories of technicolor," Nucl. Phys. B 175, 197 (1980).

[27] E. Witten, "An SU(2) anomaly," Phys. Lett. B 117, 324 (1982).

[28] A. N. Redlich, "Gauge noninvariance and parity nonconservation of three-dimensional fermions," Phys. Rev. Lett. 52, 18 (1984); "Parity violation and gauge noninvariance of the effective gauge field action in threedimensions," Phys. Rev. D 29, 2366 (1984).

[29] U. Magnea, "Three-dimensional QCD in the adjoint representation and random matrix theory," Phys. Rev. D 62, 016005 (2000) arXiv:hep-th/9912207.

[30] R. Gilmore, "Lie Groups, Lie Algebras, and Some of Their Applications," John Wiley \& Sons (New York, 1974). 